\title{
METÁSTASIS SUPRARRENAL DE SARCOMA DE EWING. A PROPÓSITO DE UN CASO
}

\author{
J. RODRÍGUEZ CORCHERO, R.A. MEDINA LÓPEZ, R. BARRERO CANDAU, \\ E. AGÜELLES SALIDO, M.P. PÉREZ ESPEJO, J.L. PASCUAL DEL POBIL MORENO \\ Servicio de Urología. Hospital Universitario Virgen del Rocío. Sevilla.
}

Actas Urol Esp. 28 (1): 70-74, 2004

\section{RESUMEN}

METÁSTASIS SUPRARRENAL DE SARCOMA DE EWING. A PROPÓSITO DE UN CASO

Los tumores metastásicos suprarrenales son más frecuentes que el carcinoma suprarrenal primario. Por orden de frecuencia se detectan metástasis de carcinomas de mama y pulmón, adenocarcinomas renal y gástrico, carcinoma de glándula suprarrenal contralateral y melanoma. Menos frecuentes son las de carcinomas de vejiga, colon, esófago, vesícula biliar, hígado, páncreas, próstata, estómago y útero. El resto de los tumores raramente se diseminan hacia las glándulas suprarrenales.

La afectación metastásica de la glándula suprarrenal por tumores de estirpe sarcomatosa es excepcional y suele darse en el contexto de una enfermedad diseminada con metástasis multiorgánicas.

Presentamos el caso de un paciente con metástasis suprarrenal de sarcoma de Ewing que nos es remitido para tratamiento quirúrgico con intención curativa, al tratarse de una metástasis única, ya que no se objetivan signos de enfermedad en otros órganos en el momento de hallarse la citada lesión.

PALABRAS CLAVE: Sarcoma de Ewing. Metástasis. Glándula suprarrenal.

\section{ABSTRACT}

\section{ADRENAL METASTASES OF EWING'S SARCOMA. A PROPOS OF ONE CASE}

Adrenal metastases are more frecuent than primary adrenal carcinoma. The most frequent are, in this order: breast and lung cancer, stomach and kidney carcinomas, adrenal gland contralateral tumors and melanoma. Less frequent are tumours of the bladder, colon, esophagus, gall bladder, liver, pancreas, prostate, stomach and uterus. The rest of the tumours rarely spread toward adrenal glands.

The metastases to the adrenal glands caused by sarcomas are rare and normaly they happen in the constest of a masive metastases afecting several organs at the same time.

We present a case of a patient affected by adrenal metastases of Ewing's sarcoma who has been referred to us for surgical treatment because it is a unique metastases (there is no sing of more illnesses in other organs at the time of the exploration). 
$\mathrm{E}^{1}$ tumor de Ewing es un sarcoma maligno compuesto por células redondas de pequeño tamaño, que suele aparecer en las tres primeras décadas de la vida. La mayoría se produce en huesos largos, aunque puede afectar a todos los demás. Es extraordinariamente maligno y la tasa de curación mediante cirugía ablativa es reducida. Es muy radiosensible, teniendo también cierta respuesta a los tratamientos quimioterápicos. Metastatiza fundamentalmente en pulmón, otros huesos, pleura y ganglios linfáticos mesentéricos.

Son muy pocos los casos documentados de metástasis suprarrenal de este tumor, y generalmente se producen en casos de enfermedad diseminada, con múltiples implantes en diferentes órganos.

\section{CASO CLÍNICO}

Paciente varón de 25 años remitido a nuestras consultas por el Servicio de Oncología Médica al presentar una imagen sugestiva de metástasis suprarrenal en una Tomografía Axial Computerizada (TAC) de control realizada para seguimiento de su enfermedad de base (Sarcoma de Ewing).

\section{Antecedentes personales}

- No presenta alergias conocidas.

- Asma bronquial extrínseca, actualmente asintomático sin tratamiento.

- Amigdalectomizado.

- Fracturas craneales en dos ocasiones (al año y a los 9 años de edad).

- En 1991 es diagnosticado de sarcoma de Ewing en pala ilíaca derecha no metastásico. Remisión completa en 1992 tras tratamiento con radio-quimioterapia pélvica.

- Tras encontrarse asintomático durante 6 años, aparece en TAC de control (1998) una imagen en mediastino anterosuperior sugestiva de recidiva. Se realiza videotoracoscopia y biopsia confirmándose la recidiva mediastínica por lo que se inicia nuevo tratamiento quimioterápico, con respuesta favorable (segunda remisión completa).

- En 1999 se decide realizar un autotrasplante de médula ósea como tratamiento de consolidación.

- En febrero de 2001 aparecen en una radio- grafía de tórax de control dos nódulos en lóbulo superior derecho, por lo que se realiza toracotomía y resección atípica de los mismos con linfadenectomía, siendo el resultado del estudio anatomopatológico de metástasis de sarcoma de Ewing.

\section{Historia actual}

Paciente con los antecedentes antes referidos, que llega a nuestras consultas por objetivarse en una TAC de control una imagen sugestiva de metástasis de su enfermedad en glándula suprarrenal izquierda. Dicha imagen no aparecía en un control realizado cuatro meses antes.

Tras solicitar punción-aspiración con aguja fina (PAAF) de dicha masa suprarrenal se confirma por estudio citológico la sospecha de metástasis.

\section{Exploración fisica}

Paciente con marcada delgadez que presenta buen estado general objetivo y buena coloración cutáneo-mucosa. Se encuentra consciente, orientado, afebril y estable hemodinámicamente.

Auscultación Cardiorespiratoria (ACR): corazón rítmico a $85 \mathrm{spm}$, sin soplos ni extratonos. TA: 90/60 mmHg. Buena ventilación en ambos hemitórax.

Abdomen: blando, depresible, sin dolor. No se palpan masas ni organomegalias.

Extremidades: no edemas periféricos, musculatura conservada, no signos de insuficiencia venosa.

\section{Pruebas complementarias}

P. de laboratorio: bioquímica sanguínea, hemograma y coagulación sin alteraciones relevantes.

P. de Imagen:

1. Rx de tórax: pérdida de volumen en lóbulo superior derecho (LSD), existencia de tractos fibrosos y atelectasia cicatricial asociada a engrosamiento pleural adyacente, lo que sugiere la existencia de un proceso específico en fase de cicatrización.

2. TAC toraco-abdominal: destaca una pérdida de volumen en hemitórax derecho a expensas del lóbulo superior con tractos lineales hiperdensos que se extienden hacia el vértice (Fig. 1a) e 
impresionan como de aspecto fibroso-cicatricial. Proyectándose sobre el polo superior del riñón izquierdo, visualizamos una imagen nodular de baja densidad (Fig. 1b), bien delimitada y de aprox. $4 \mathrm{~cm}$ de diámetro, con densidad ligeramente superior a la del líquido, por lo que pudiera corresponder a un quiste complicado, sin poder descartar la posibilidad de lesión metastásica.

\section{Tratamiento}

Tras completar los estudios preanestésicos se interviene quirúrgicamente $(1 / 7 / 02)$, realizándose suprarrenalectomía izquierda mediante lumbotomía (Fig. 2).

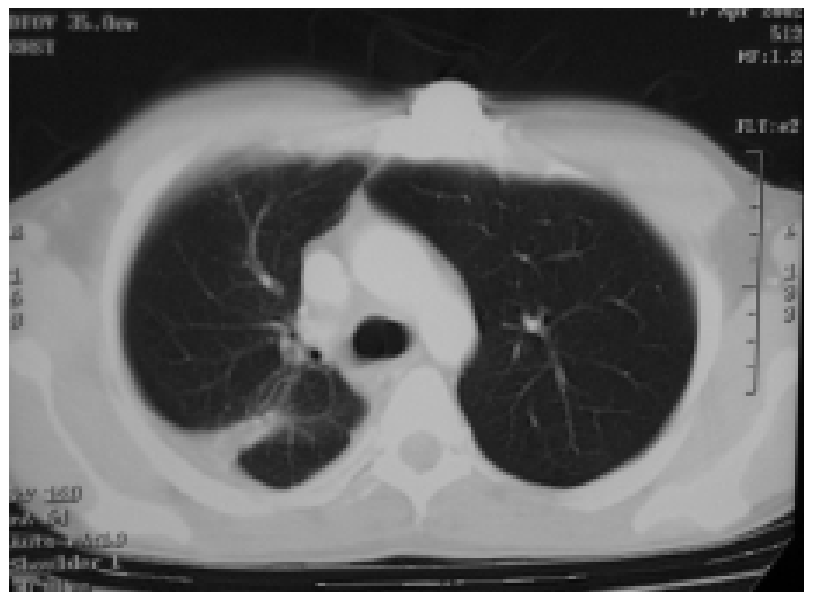

FIGURA 1a. TAC toraco-abdominal: condensación de lóbulo superior de pulmón derecho con aspecto fibroso-cicatricial.

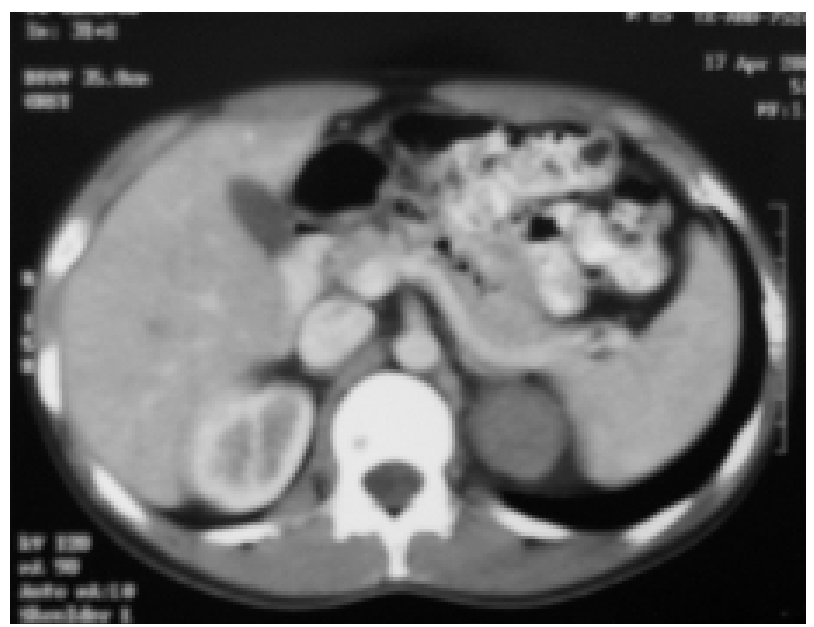

FIGURA 1b. TAC toraco-abdominal: imagen nodular de 4 cm de diámetro sugerente de metástasis suprarrenal o quiste complicado.

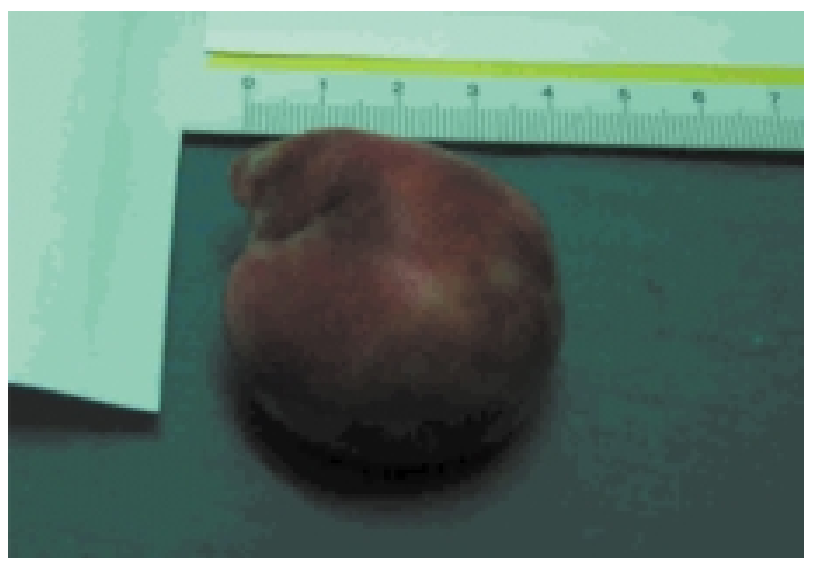

FIGURA 2. Aspecto macroscópico de la pieza quirúrgica.

\section{Evolución}

Favorable, siendo dado de alta hospitalaria a la semana de la intervención quirúrgica, estando asintomático.

Revisado en nuestras consultas externas, presenta herida quirúrgica con buen aspecto, confirmándose con la anatomía patológica de la pieza quirúrgica la presencia de metástasis de su proceso neoplásico (Figs. 3a y 3b).

Actualmente el paciente está siendo revisado por el Servicio de Oncología Médica, siendo dado de alta por parte de Urología.

\section{COMENTARIOS}

El sarcoma de Ewing es el segundo tumor óseo maligno en frecuencia de la infancia. Representa un 3-6\% de todos los tumores sólidos y el 1,4-1,8\% de los procesos malignos en individuos de raza blanca menores de 15 años, donde la incidencia anual es de 3 casos por millón ${ }^{1}$.

Es más frecuente en varones, afectando preferentemente al esqueleto axial, aunque puede afectar a cualquier hueso. Las localizaciones más frecuentes son: pelvis (20\%), fémur (17\%) y húmero (10\%).

Además del dolor habitual de los tumores óseos, un tercio de los pacientes debutan con fiebre, leucocitosis, velocidad de sedimentación globular (VSG) elevada y signos locales que sugieren erróneamente un proceso infeccioso.

La vía de diseminación más común es la hematógena, siendo las metástasis más frecuentes las localizadas en pulmón (57\%), huesos, médula ósea, pleura y ganglios linfáticos. 


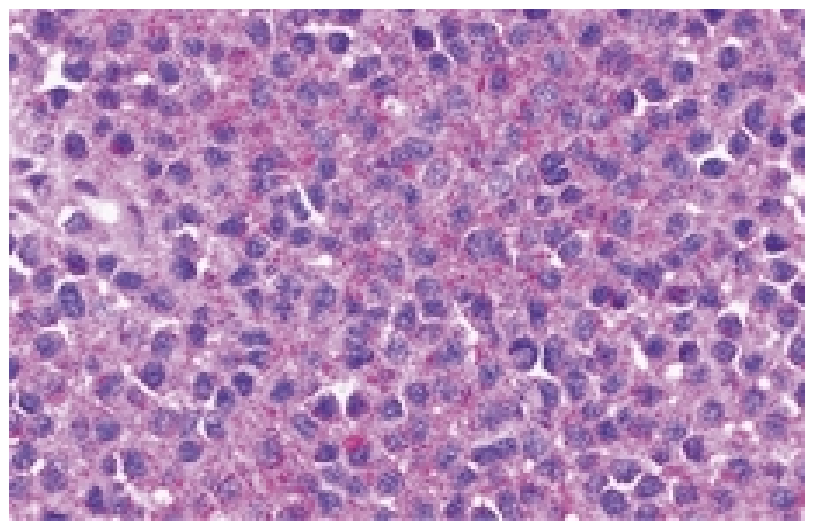

FIGURA 3a. Sarcoma de Ewing. Tinción de PAS.

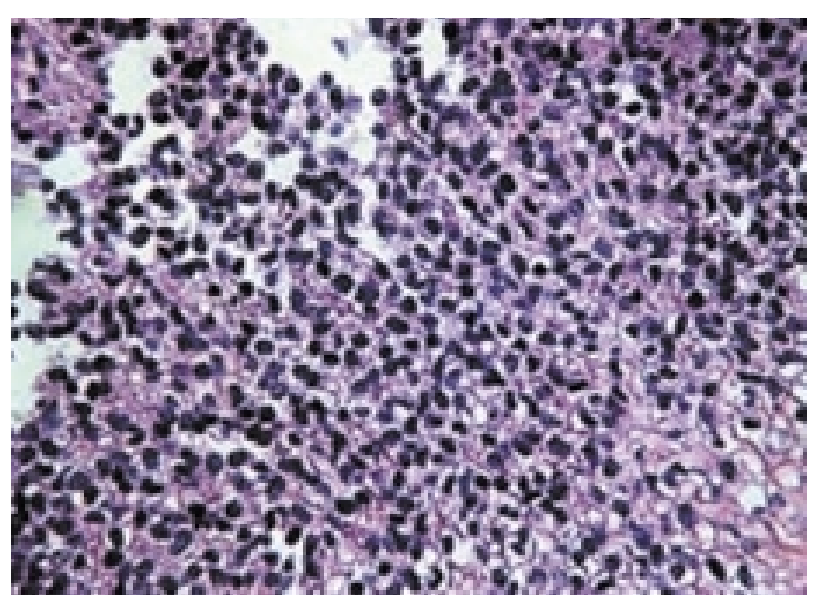

FIGURA 3b. Sarcoma de Ewing. Aspecto tipico: acúmulos sólidos de células separadas por finas bandas. Las células son redondeadas, uniformes, con escotaduras nucleares $y$ nucleolo visible.

Para el diagnóstico se utilizan la radiografía simple en la que a veces puede simular una lesión benigna ${ }^{2}$, pruebas de laboratorio (elevación de VSG y LDH) y fundamentalmente el TAC, que es el mejor método convencional para el estadiaje local y a distancia. No obstante, el diagnóstico definitivo es anatomopatológico, caracterizándose al microscopio óptico por acúmulos sólidos de células separadas por finas bandas. Las células son redondeadas, uniformes, con escotaduras nucleares y nucleolo visible. El citoplasma es claro, conteniendo glucógeno (PAS+) ${ }^{1}$.

En cuanto al tratamiento, debe considerarse una enfermedad de carácter sistémico desde el momento del diagnóstico. El control local se basa en un tratamiento multidisciplinario combinando cirugia, quimio y radioterapia (es un tumor muy radiosensible). Para el control sistémico se usa poliquimioterapia cíclica asociada o no a radioterapia $^{3,4}$.

El pronóstico viene determinado por el grado de indiferenciación del tumor, modo de diseminación y tiempo hasta la recidiva desde la remisión completa (si <24 meses, indica peor pronóstico) ${ }^{4}$.

En cuanto a las metástasis suprarrenales, en general, son raras, aunque más frecuentes que el carcinoma suprarrenal primario. La mayoría se diagnostican en los primeros diez meses tras el diagnóstico del tumor primario. El 90\% son de carcinomas, principalmente de pulmón, mama, estómago, riñón y glándula suprarrenal contralateral $^{5}$. Menos frecuentes son las metástasis de tumores de vejiga, colon, esófago, vía biliar, hígado, páncreas, próstata y útero. Otro tumor que metastatiza con cierta frecuencia en las glándulas suprarrenales es el melanoma. El resto de los tumores no se diseminan hacia las glándulas suprarrenales, salvo en casos excepcionales, como el que nos ocupa.

Hasta en un $50 \%$ de los casos las metástasis son bilaterales, siendo las unilaterales más frecuentes en el lado izquierdo ${ }^{6,7}$.

Para el diagnóstico, las técnicas de elección son la ecografía y principalmente la TAC, por su mayor especificidad ${ }^{7,8}$. La PAAF está indicada en los casos en los que sea importante la confirmación histológica de la metástasis para plantear el tratamiento más oportuno, no siendo necesaria en todas las masas suprarrenales ${ }^{9}$.

En lo que concierne al tratamiento de las metástasis suprarrenales, éste depende de la histología del tumor primario y de la situación en que se encuentre la enfermedad. En caso de metástasis únicas, suele optarse por la cirugía radial (abierta o por vía laparoscópica) ${ }^{5-7,10,11}$, si bien depende del tratamiento de elección para el tumor primitivo.

La singularidad de este caso clínico, radica en que hay muy pocos casos recogidos en la literatura de metástasis suprarrenal de tumores óseos ${ }^{12,13}$, y los que hay se refieren a pacientes con metástasis multiorgánicas en el momento del diagnóstico de la localizada en glándula suprarrenal y, por lo tanto, no subsidiarios de tratamiento quirúrgico. 


\section{REFERENCIAS}

1. PAULUSSEN M, FROHLICH B, JURGENS H.: Ewing tumor: incidence, prognosis and treatment options. Paediatr Drugs 2001; 3 (12): 899-913.

2. MILLER SL, HOFFER FA.: Malignant and benign bone tumors. Radiol Clin North Am 2001 jul; 39 (4): 673-699.

3. MEYERS PA, LEVY AS.: Ewing's sarcoma. Curr Treat Options Oncol 2000 aug; 1 (3): 247-257.

4. BURDACH S, JURGENS H.: High-dose chemoradiotherapy in the Ewing family tumors. Crit Rev Oncol Hematol 2002 feb; 41 (2): 169-189.

5. LAM KY, LO CY.: Metastatic tumours of the adrenal glands: a 30-year experience in a teaching hospital. Clin Endocrinol (Oxf) 2002 jan; 56 (1): 95-101.

6. SALINAS SÁNCHEZ A, LORENZO ROMERO J, SEGURA MARTÍN M, HERNÁNDEZ MILLÁN I, PASTOR GUZMÁN J, VIRSEDA RODRÍGUEZ J.: Patología quirúrgica suprarrenal. 10 años de experiencia y revisión de la literatura. Arch Esp Urol 1998 apr; 51 (3): 327-340.

7. HENIFORD BT, ARCA MJ, WALSH RM, GILL IS. Laparoscopic adrenalectomy for cancer. Semin Surg Oncol 1999 jun; 16 (4): 293-306.

8. LOCKHART ME, SMITH JK, KENNEY PJ.: Imaging of adrenal masses. Eur J Radiol 2002 feb; 41 (2): 95112 .
9. LEE JE, EVANS DB, HICKEY RC, SHERMAN SI, GAGEL RF, ABBRUZZESE MC, ABBRUZZESE JL.: Unkown primary cancer presenting as an adrenal mass: frequency and implications for diagnostic evaluation of adrenal incidentalomas. Surgery 1998 dec; 124 (6): 1115-1122.

10. SUNG GT, GILL IS.: Laparoscopic adrenalectomy. Semin Laparosc Surg 2000 sep; 7 (3): 211-222.

11. KATAYAMA A, MAFUNE K, MAKUUCHI M.: Adrenalectomy for solitary adrenal metastasis form colorectal carcinoma. Jpn Clin Oncol 2000 sep; 30 (9): 414-416.

12. POTEPAN P, DANESINI GM, SPAGNOLI I, LAFFRANCHI A, GIOVANNARDI G, TURRINI E, GUZZON A.: Adrenal gland metastasis in osteogenic osteosarcoma. A radiological case report. Tumori 1992 dec 31; 78 (6): 417-420.

13. ZIDKOVA H, DRECHSLEROVA J, MATEJOVSCY Z, KASPAR M, KOLAR J.: Extensive metastases of Ewing's sarcoma in the adreno-renal area. Cesk Radiol 1990 jan; 44 (1): 37-40.

Dr. J. Rodríguez Corchero

Servicio de Urología. Hosp. Univ. Virgen del Rocío Avda. Manuel Siurot, s/n

41013 Sevilla

(Trabajo recibido el 27 mayo de 2003) 